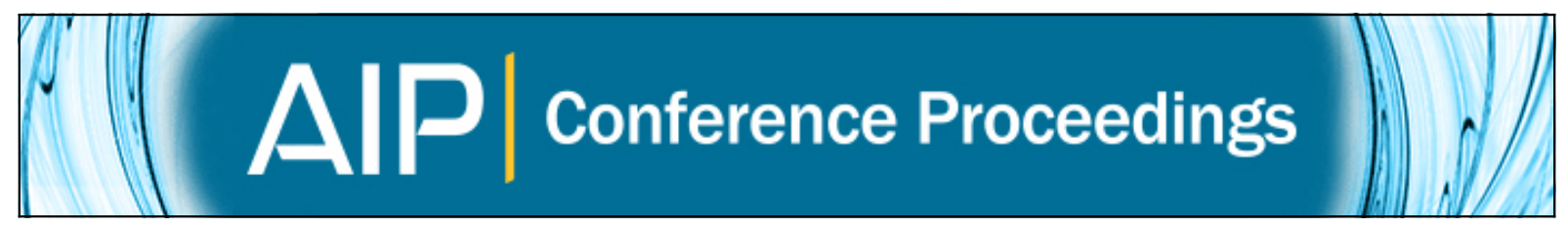

\title{
Exclusion of Tiny Interstellar Dust Grains From the Heliosphere
}

J. D. Slavin, P. C. Frisch, J. Heerikhuisen, N. V. Pogorelov, H.-R. Mueller, W. T. Reach, G. P. Zank, B. Dasgupta , and K. Avinash

Citation: AIP Conference Proceedings 1216, 497 (2010); doi: 10.1063/1.3396301

View online: http://dx.doi.org/10.1063/1.3396301

View Table of Contents: http://scitation.aip.org/content/aip/proceeding/aipcp/1216?ver=pdfcov

Published by the AIP Publishing

\section{Articles you may be interested in}

Modeling outer heliosphere structures near the heliopause

AIP Conf. Proc. 1539, 360 (2013); 10.1063/1.4811061

Relating IBEX and Voyager Data through Global Modeling of the Heliospheric Interface

AIP Conf. Proc. 1302, 3 (2010); 10.1063/1.3529988

Whistlers in interstellar gas dynamics

AIP Conf. Proc. 609, 102 (2002); 10.1063/1.1471831

Interaction of evolved pulsars and magnetars with the ISM

AIP Conf. Proc. 586, 519 (2001); 10.1063/1.1419603

Heliospheric interface filtration of the interstellar hydrogen

AIP Conf. Proc. 471, 803 (1999); 10.1063/1.58828 


\title{
Exclusion of Tiny Interstellar Dust Grains From the Heliosphere
}

\author{
J. D. Slavin*, P. C. Frisch ${ }^{\dagger}$, J. Heerikhuisen**, N. V. Pogorelov**, H.-R. Mueller ${ }^{*}$, \\ W. T. Reach ${ }^{\S}$, G. P. Zank ${ }^{* *}$, B. Dasgupta** and K. Avinash** \\ *Harvard-Smithsonian Center for Astrophysics, 60 Garden St., Cambridge, MA 02138 (USA) \\ ${ }^{\dagger}$ Dept. Astronomy \& Astrophysics, U. Chicago, 5640 S. Ellis Ave., Chicago, IL 60637 (USA) \\ ${ }^{* *}$ U. Alabama-Huntsville, Center for Space Plasma and Aeronomic Research, Huntsville, AL 35899 \\ $\$$ Dept. of Physics and Astronomy, 6127 Wilder Lab, Dartmouth College, Hanover, NH 03755 \\ $\S$ Infrared Processing and Analysis Center, Caltech, Pasadena, CA 91125
}

\begin{abstract}
The distribution of interstellar dust grains (ISDG) observed in the Solar System depends on the nature of the interstellar medium-solar wind interaction. The charge of the grains couples them to the interstellar magnetic field (ISMF) resulting in some fraction of grains being excluded from the heliosphere while grains on the larger end of the size distribution, with gyroradii comparable to the size of the heliosphere, penetrate the termination shock. This results in a skewing the size distribution detected in the Solar System.

We present new calculations of grain trajectories and the resultant grain density distribution for small ISDGs propagating through the heliosphere. We make use of detailed heliosphere model results, using three-dimensional (3-D) magnetohydrodynamic/kinetic models designed to match data on the shape of the termination shock and the relative deflection of interstellar $\mathrm{H}^{\mathrm{o}}$ and $\mathrm{He}^{\mathrm{O}}$ flowing into the heliosphere. We find that the necessary inclination of the ISMF relative to the inflow direction results in an asymmetry in the distribution of the larger grains $(0.1 \mu \mathrm{m})$ that penetrate the heliopause. Smaller grains $(0.01$ $\mu \mathrm{m})$ are completely excluded from the Solar System at the heliopause.
\end{abstract}

Keywords: heliosphere; interstellar matter

PACS: $96.50 . \mathrm{Xy}, 95.30 . \mathrm{Wi}, 96.50 . \mathrm{Dj}, 96.50 . \mathrm{Ek}, 98.38 . \mathrm{Cp}$

\section{INTRODUCTION}

Up to one percent of the mass of the interstellar cloud surrounding the heliosphere is carried by dust grains that interact with the heliosphere [1,2]. The flow of interstellar material past the Sun at $26.4 \mathrm{~km} \mathrm{~s}^{-1}$ drives large interstellar dust grains into the heliosphere, while small grains are diverted around the heliosphere by the interstellar magnetic field (ISMF). The heliospheric trajectories of intermediate sized grains can be complicated and depend on the solar wind magnetic field and thus solar cycle phase. Observations of ISDGs in the solar system by the Ulysses, Galileo and Cassini spacecraft show that the density of smallest grains are deficient in the inner heliosphere when compared to the nominal Mathis et al. "MRN" power law size distribution [e.g. 3, 4]. For instance, the density of grains of mass $10^{-14} \mathrm{~g}(a \sim 0.1$ $\mu \mathrm{m})$ is reduced in the inner heliosphere by a factor of $\sim 90$ below the MRN predictions and $10^{-15} \mathrm{~g}(a \sim 0.05$ $\mu \mathrm{m})$ grains are deficient by three orders of magnitude. At the same time large $(a \sim 1 \mu \mathrm{m})$ grains are absent from the MRN distribution, but are abundant in the inflowing ISDGs. While several alternative grain size distribution models exist [e.g., 5, 6, 7], the constraints of staying within the limits of cosmic abundances and explaining the interstellar extinction curve lead in all cases to the presence in the models of small grains that are absent from the observed distribution and a cutoff on the large grain size end that is below that of the observed distribution. The deficiencies of small grains may result from small grains being deflected around the heliosphere because their high charge-to-mass ratios cause them to couple tightly to the ISMF. On the other hand, the circumheliospheric interstellar medium (CHISM) may simply be deficient in small grains (balancing the overabundance of large grains). To evaluate which is the case requires calculations of the grain distribution as a function of grain size in the context of realistic heliosphere models. With recent data on the shape of the heliosphere from the crossing of the termination shock (TS) by Voyagers 1 and 2 [8], and on the deflection of inflowing interstellar $\mathrm{H}^{0}$ relative to $\mathrm{He}^{0}$ [9], such models face tighter constraints than ever before. The inferred asymmetry of the heliosphere can be explained by the orientation of the ISMF relative to the direction of the cloud-Sun relative motion. The ISMF required to fit the heliosphere data, in turn, has direct implications for ISDG trajectories.

In this paper we present new calculations of grain trajectories and density distributions for small grains that are primarily excluded from the inner heliosphere. Small grains experience enhanced charging rates due to the ejection of secondary electrons in the hot $10^{5}-10^{6} \mathrm{~K}$ 
plasma between the termination shock and heliopause [10]. We calculate grain trajectories in 3-D, based on a self-consistent steady state MHD-kinetic heliosphere model with grain charge calculated at each location along the path according to the plasma conditions and radiation field. (We note that the recent Voyager 2 results [11] on the lower than expected plasma temperature in the inner heliosheath are not incorporated in the heliosphere model we use. We expect that this could result in somewhat lower dust charging in this region than we calculated, though the detailed effects of charging by thermal particles vs. PUIs has yet to be fully explored.) The model of plasma density and magnetic field in the heliosphere at each point of space properly accounts for the asymmetries that result from the angle between the ISMF and interstellar gas/dust inflow direction, and ISMF and ecliptic plane $[12,13]$. When combined with recent models of grain charging [14] and a realistic ultraviolet (UV) radiation field, the Lorentz force at each point in space can be used to calculate the grain trajectory. Total 3-D grain densities are then calculated for a sample of interstellar grain sizes based on the total gas-to-dust mass ratio in the CHISM and ISDG models. Many previous calculations have been made of grain interactions with the heliosphere [e.g. 15, 3, 10, 2] but ours is the first to use a realistic heliosphere model, including distortion due to an ISMF orientation that is consistent with recent observational data.

\section{GRAIN TRAJECTORY CALCULATIONS}

\section{Heliosphere Model}

The grain trajectory calculations require a 3-D model that self-consistently includes the interaction and charge exchange between interstellar $\mathrm{H}$ and the solar wind plasma, and that is based on realistic boundary conditions for the heliosphere as set by the surrounding interstellar cloud. The $10 \mathrm{AU}$ difference in the termination shock distances seen by Voyager 1 versus Voyager 2 suggests an asymmetry in the outer heliosphere topology that can be explained by the tilt of the ISMF with respect to the upwind direction. We therefore select a heliosphere model that reproduces the TS asymmetry, the hydrogenhelium offset of $\sim 5^{\circ}$, and the angle of the ISMF with respect to the ecliptic plane as indicated by the H-He offset and data on the ISMF near the Sun. We use the heliosphere of Pogorelov et al. [12, 13] and Heerikhuisen et al. [16], which is based on coupled 3-D MHD (ion) fluid and kinetic (neutral) particle code including the effects of charge exchange to calculate the plasma and neutral particle properties. The model parameters of the solar wind and inflowing interstellar material are given in Ta-

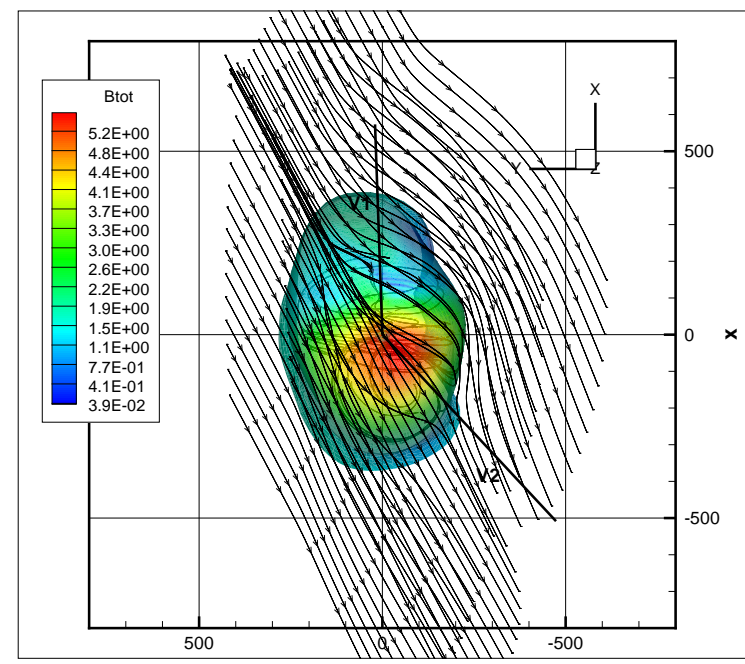

FIGURE 1. 3-D MHD/kinetic heliosphere model used in these calculations [12, 13], showing the heliopause boundary, magnetic field lines and the Voyager 1 and 2 trajectories. The viewpoint is from interstellar space looking towards the downwind. The heliopause is colored according to the strength of the magnetic field. The bending of the magnetic field lines around the heliopause dominates the trajectories of tiny dust grains interacting with the heliosphere, and is responsible for the dust trajectories and hence the enhanced dust densities.

ble 1 (where $n\left(\mathrm{H}^{0}\right)$ is the neutral $\mathrm{H}$ density and $n\left(\mathrm{p}^{+}\right)$is the proton density). The solar wind in the model carries a Parker spiral type magnetic field and has $v\left(\mathrm{H}^{+}\right)_{\mathrm{SW}}=450$ $\mathrm{km} \mathrm{s}^{-1}$. The ISMF strength is $B_{\mathrm{ISM}}=3 \mu \mathrm{G}$ with the direction $30^{\circ}$ away from the ISM inflow direction and pointed toward the southern hemisphere, and lying in the $\mathrm{H}$ deflection plane for this model [13]. The heliosphere model uses a coordinate system with the $\mathrm{x}$-axis perpendicular to the ecliptic plane, the $\mathrm{z}$-axis in the ecliptic plane towards upwind and perpendicular to the $\mathrm{x}$-axis, and the $y$-axis in the ecliptic plane to form a righthanded coordinate system. The Sun is located at $x=y=z=0$. Fig. 1 shows the ISMF lines bending over the heliopause.

\section{Grains and Grain Charging}

We illustrate the deflection of small grains around the heliosphere by calculating the charge and trajectories for spherical grains of radii $0.1 \mu \mathrm{m}$, and $0.01 \mu \mathrm{m}$, composed of astronomical silicates. The assumption that the small grains are silicates is supported by observations and analysis indicating that the gas phase carbon abundance in the CHISM is high [supersolar in fact 17] leaving none to be locked up in grains. Grain charging is calculated using the model and grain-charging code of Weingartner et al. [14], combined with plasma densities and temperatures from the heliosphere model, and the modeled extreme- 
TABLE 1. Parameters of Heliosphere Model with Positive Magnetic Polarity at the NEP

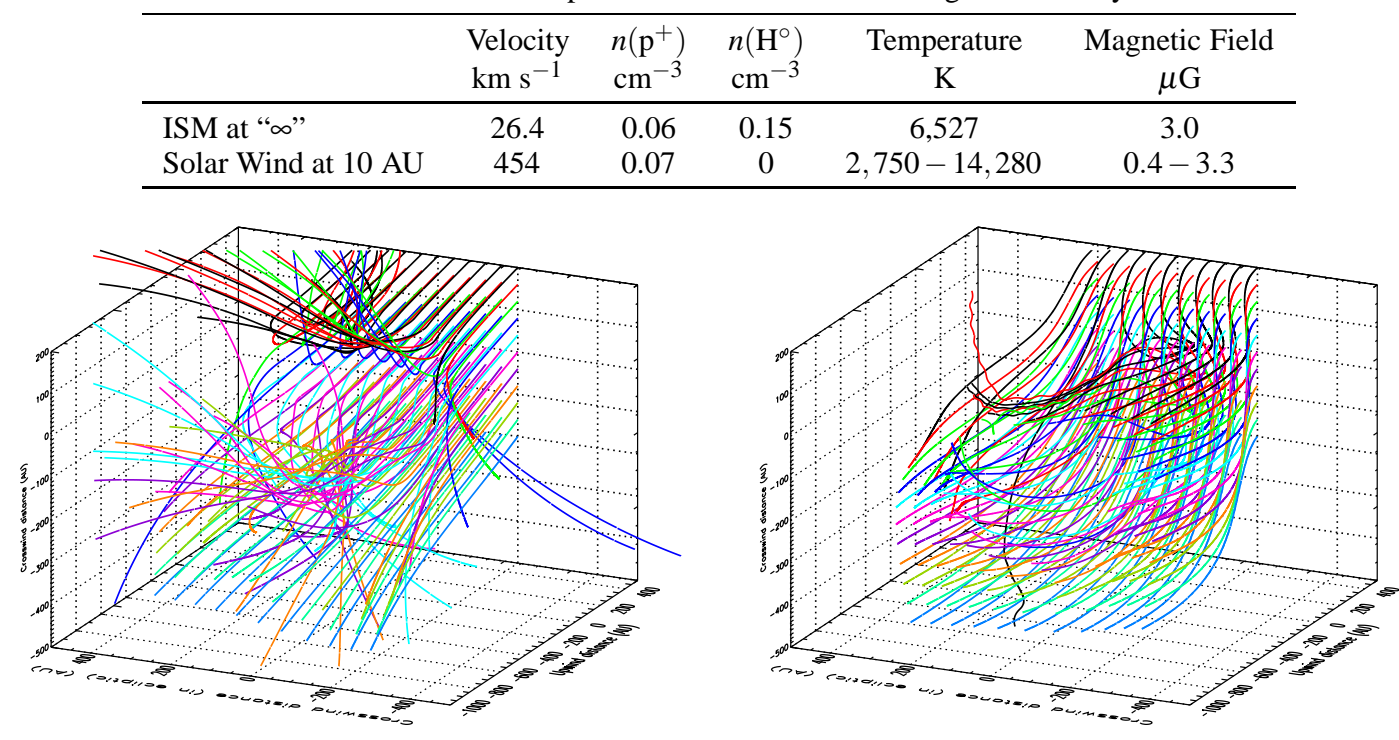

FIGURE 2. Example of spatial trajectories for $0.1 \mu \mathrm{m}$ grains (left) and $0.01 \mu \mathrm{m}$ grains (right). The upwind direction is toward the back plane while the ecliptic is parallel to the bottom plane. Note the convergence of trajectories in the top and bottom, with most $0.01 \mu \mathrm{m}$ grains deflected to low latitudes. The $0.01 \mu \mathrm{m}$ grains are entirely deflected around the heliosphere.

UV/far-UV photon fluxes as discussed below.

The primary mechanisms affecting the charge of grains near and in the heliosphere are the photoelectric emission caused by absorption of photons from the UV radiation field, and the sticking of electrons and ions that collide with the grains. Additional grain charging occurs in the hot subsonic solar wind plasma in the inner heliosheath between the solar wind termination shock and the heliopause because of secondary electron emission produced by collisions of high energy electrons with the grains.

The radiation field used in our calculations is comprised of the constant interstellar radiation field from Gondhalekar et al. [18], and the Solar flux data taken from the TIMED/SEE project (see http://lasp. colorado.edu/see/see_data.html). The TIMED/SEE project creates spectra using a combination of measured and modeled fluxes. The photon flux longward of $13.6 \mathrm{eV}$ is dominated by the interstellar radiation field, while the extreme ultraviolet radiation field is dominated by the Sun. The interstellar and solar fluxes have equal effect in charging grains at $\approx 100 \mathrm{AU}$.

\section{Grain Trajectories}

The grain trajectory calculations begin with the grains upstream of the heliosphere and traveling with the interstellar gas. However, the grains are likely to have been accelerated by turbulence in the interstellar magnetic field and thus should have some initial velocity relative to the gas. Taking guidance from Yan et al. [19], we give the grains an initial $3 \mathrm{~km} \mathrm{~s}^{-1}$ speed (roughly the gas turbulent velocity observed for the Local Interstellar Cloud) relative to the gas, and with a net direction that is perpendicular to the magnetic field but is otherwise randomly directed.

We integrate the equations of motion for the grains, calculating at each point the charge on the grain depending on its position (and properties of the surrounding plasma) and including the Lorentz forces, radiation pressure and gravity of the Sun. For the density distribution we take over $10^{6}$ grain trajectories for grains starting in a grid in a plane far $(\sim 900 \mathrm{AU})$ from the Sun in the ISM.

The forces acting on the grains include gravity, radiation pressure, and the Lorentz force from the motion of charged grains relative to the magnetic field lines. The equation of motion is given by

$$
\ddot{\vec{x}}_{\mathrm{g}}+(1-\beta) \frac{G M_{\text {sun }}}{\left|\vec{x}_{\mathrm{g}}\right|^{3}} \vec{x}_{\mathrm{g}}-\frac{q}{m}\left(\left(\dot{\vec{x}}_{\mathrm{g}}-\vec{v}_{\mathrm{gas}}\right) \times \vec{B}\right)=0
$$

where $\dot{\vec{x}}_{\mathrm{g}}, q$, and $m$ are the velocity, charge and mass of the grain, $\beta$ is the ratio of radiation pressure to gravitational attraction, $\vec{B}$ is magnetic field, and $\vec{v}_{\text {gas }}$ is gas (solar wind or ISM) velocity. Although radiation pressure is not significant for spherical grains in the outer heliosheath that obey Mie scattering, Kimura and Mann [20] have shown that radiation pressure acting on fluffy grains results in non-radial torques that may significantly perturb 

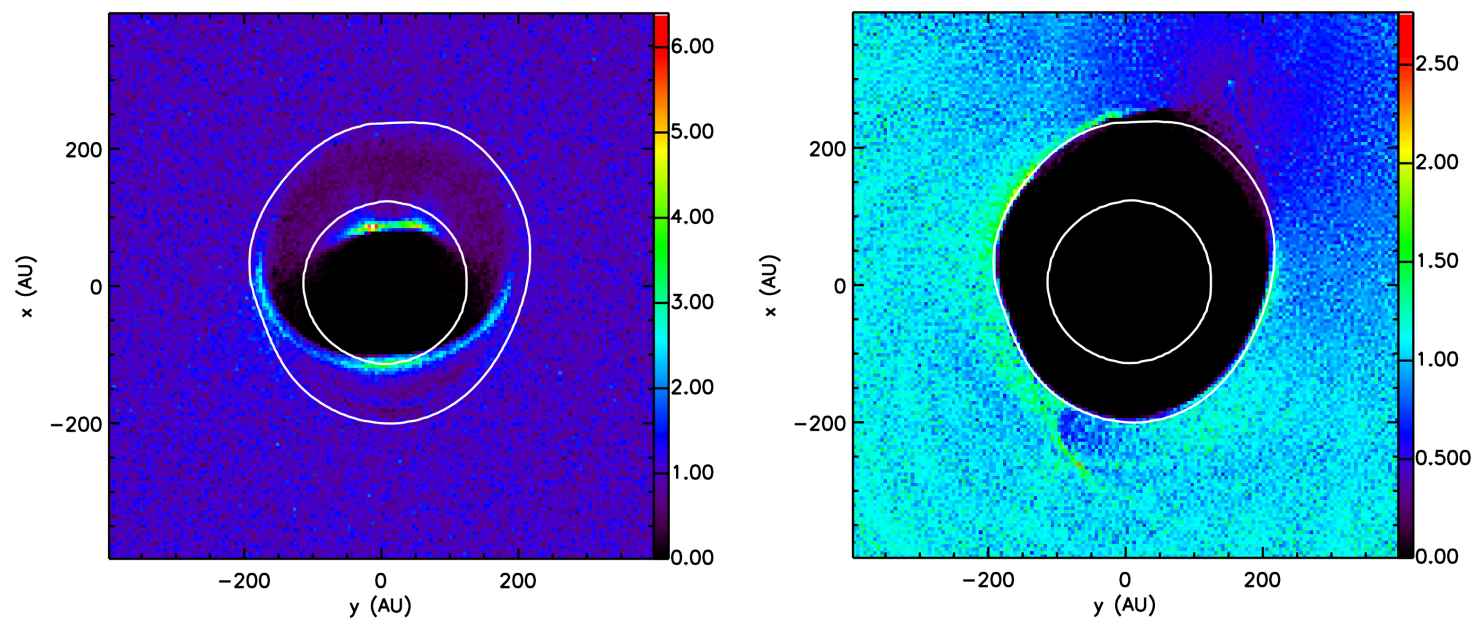

FIGURE 3. Dust density distributions $\left(\mathrm{cm}^{-3}\right)$ in a slice in the $\mathrm{z}=0$ plane (perpendicular to the IS flow direction) for radius $0.1 \mu \mathrm{m}$ (left figure) and $0.01 \mu \mathrm{m}$ (right figure) silicate grains. (See text for coordinate system.) These views are centered near the upwind direction, and ecliptic longitude increases towards the left. The inner nearly circular contour is the solar wind termination shock, while the outer contour is the heliopause. The $a \sim 0.1 \mu \mathrm{m}$ grains are partially diverted at the heliopause, but some grains penetrate inside the TS. All $0.01 \mu \mathrm{m}$ grains, which have small gyroradii due to a high $q / \mathrm{m}$ ratio, are excluded from the heliosphere. The colors indicate the dust density relative to its value in the pristine ISM. Note that the plotted density range is much larger for the $0.1 \mu \mathrm{m}$ grains.
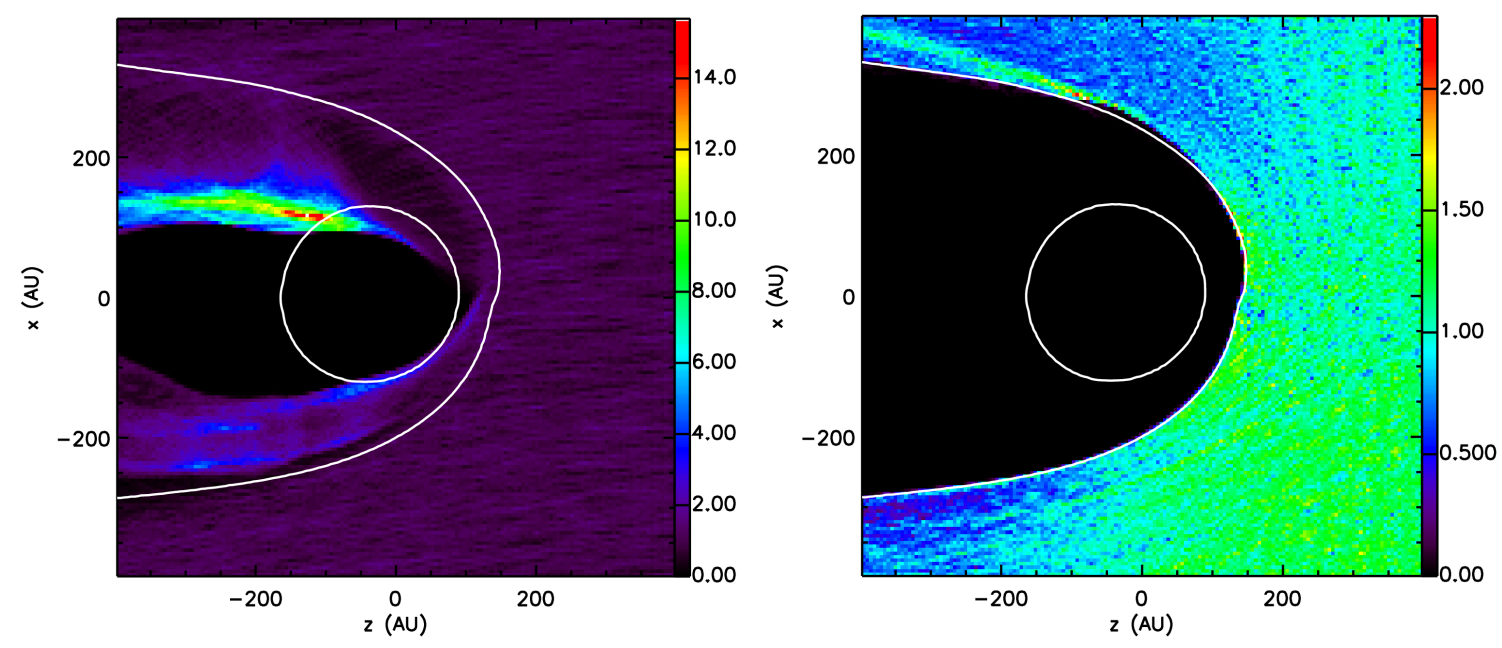

FIGURE 4. Spatial densities of grains $\left(\mathrm{cm}^{-3}\right)$ for slices in the $\mathrm{xz}$ plane. The right figure $(0.01 \mu \mathrm{m}$ grains $)$ shows the full exclusion of tiny grains from the heliosphere. The left figure $(0.1 \mu \mathrm{m}$ grains) shows the diversion of slightly larger grains able to partially penetrate the inner heliosheath. The concentration of grains near the North Ecliptic Pole results from the deflection of particles in the inner heliosheath where they are sensitive to the magnetic polarity of the solar wind.

the grain motion in the outer heliosphere. This effect is not included here.

We show sample grain trajectories for silicate grains of radii $0.01 \mu \mathrm{m}$ and $0.1 \mu \mathrm{m}$ in Fig. 2 . The resulting density distributions of grains $\left(\mathrm{cm}^{-3}\right)$ are shown for a slice in the plane at $z=0$ that is perpendicular to the interstellar flow direction (Fig. 3), and for a meridian slice through the heliosphere nose (Fig. 4).

These simulations of grain locations in the extended
3-D volume were performed with the University of Alabama, Huntsville, CSPAR cluster.

\section{RESULTS}

This study shows that the distortion of the local interstellar magnetic field by the heliosphere causes large-scale asymmetries in the spatial distribution of tiny interstellar 
grains flowing past the Sun and within 1000 AU. Grains of radius $\sim 0.01 \mu \mathrm{m}$ appear to be completely excluded from the heliosphere. The trajectories of these grains will be sensitive to the direction of the local interstellar magnetic field, and relatively insensitive to the solar 22-year magnetic activity cycle since the grains do not penetrate the heliopause. In Fig. 5 we show the column densities of these grains within $400 \mathrm{AU}$ of the Sun, and outside of the heliopause. The ISMF distortion by the heliosphere is imprinted on the distribution of these small grains.

Grains as small as $0.1 \mu \mathrm{m}$ can partially penetrate the termination shock. The trajectories of $0.1 \mu \mathrm{m}$ grains are initially influenced by the interstellar magnetic field. Once inside of the heliosphere, the solar wind magnetic field and solar cycle become significant [e.g. 2]. The high charge-to-mass ratio for these grains is enhanced by secondary electron ejection in the inner heliosheath, increasing the coupling between $0.1 \mu \mathrm{m}$ grains and the magnetic field in this region.

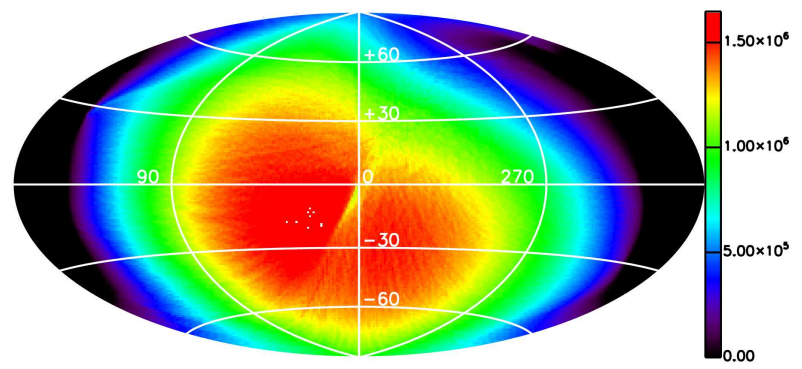

FIGURE 5. Column densities $\left(\mathrm{cm}^{-2}\right)$ of $0.01 \mu \mathrm{m}$ grains deflected around the heliopause, out to a distance of $400 \mathrm{AU}$ from the Sun. The values on the color bar show the true column density $\left(\mathrm{cm}^{-2}\right)$ for the assumptions that the gas-to-dust ratio is 100 and all the grains are $0.01 \mu \mathrm{m}$. The upwind direction is at the center, with $0^{\circ}$ corresponding to ecliptic coordinate $\ell \sim 255^{\circ}$.

\section{ACKNOWLEDGMENTS}

We thank Joe Weingartner for sharing his grain-charging code with us, and Richard Lieu, Haru Washimi, and Xianzhi Ao for helpful comments. This work has been supported by NASA grants NNX08AJ33G, NNX09AH50G, NNX07AH18G, NNX08AJ21G, NNX09AB24G, NNX09AG29G, and NNX09AG62G, and NASA contract NNG05EC85C.

\section{REFERENCES}

1. J. D. Slavin, and P. C. Frisch, $A \& A$ 491, 53-68 (2008).

2. M. Landgraf, J. Geophys. Res. 105, 10303-10316 (2000).

3. P. C. Frisch, J. M. Dorschner, J. Geiss, J. M. Greenberg,

E. Grün, M. Landgraf, P. Hoppe, A. P. Jones,

W. Krätschmer, T. J. Linde, G. E. Morfill, W. Reach, J. D.
Slavin, J. Svestka, A. N. Witt, and G. P. Zank, ApJ 525, 492-516 (1999).

4. J. S. Mathis, W. Rumpl, and K. H. Nordsieck, ApJ 217, 425-433 (1977).

5. S. Kim, P. G. Martin, and P. D. Hendry, ApJ 422, 164-175 (1994).

6. J. C. Weingartner, and B. T. Draine, ApJ 548, 296-309 (2001), arXiv:astro-ph/0008146.

7. V. Zubko, E. Dwek, and R. G. Arendt, ApJS 152, 211-249 (2004).

8. E. C. Stone, A. C. Cummings, F. B. McDonald, B. C. Heikkila, N. Lal, and W. R. Webber, Nature 454, 71-74 (2008).

9. R. Lallement, E. Quémerais, J. L. Bertaux, S. Ferron, D. Koutroumpa, and R. Pellinen, Science 307, 1447-1449 (2005).

10. H. Kimura, and I. Mann, ApJ 499, 454 (1998).

11. J. D. Richardson, J. C. Kasper, C. Wang, J. W. Belcher, and A. J. Lazarus, Nature 454, 63-66 (2008).

12. N. V. Pogorelov, E. C. Stone, V. Florinski, and G. P. Zank, ApJ 668, 611-624 (2007).

13. N. V. Pogorelov, J. Heerikhuisen, and G. P. Zank, ApJ 675, L41-L44 (2008).

14. J. C. Weingartner, B. T. Draine, and D. K. Barr, ApJ 645 , 1188-1197 (2006)

15. E. Gruen, B. Gustafson, I. Mann, M. Baguhl, G. E. Morfill, P. Staubach, A. Taylor, and H. A. Zook, $A \& A$ 286, 915-924 (1994).

16. J. Heerikhuisen, N. V. Pogorelov, V. Florinski, and G. P. Zank, "Modeling Kinetic Neutral Atoms in the Solar-Wind/Interstellar-Medium Interaction Region," in Numerical Modeling of Space Plasma Flows, edited by N. V. Pogorelov, E. Audit, and G. P. Zank, 2008, vol. 385 of Astronomical Society of the Pacific Conference Series, p. 204.

17. J. D. Slavin, and P. C. Frisch, ApJ 651, L37-L40 (2006).

18. P. M. Gondhalekar, A. P. Phillips, and R. Wilson, $A \& A$ 85, 272 (1980).

19. H. Yan, A. Lazarian, and B. T. Draine, ApJ 616, 895-911 (2004).

20. H. Kimura, and I. Mann, Journal of Quantitative Spectroscopy and Radiative Transfer 60, 425-438 (1998). 